\title{
Capturing sequences for bioprospecting
}

Recombineering between two linear molecules enables long sequences to be efficiently cloned from a genomic mixture.

The aim of bioprospecting is to mine the chemical potential of the natural world, potential that is often hidden in the genomes of organisms that cannot be seen or cultured. Although direct sequencing of environmental samples can serve as a divining rod, the shovels-PCR and library cloning-are limited to extracting short sequences and can demand laborious screening. Youming Zhang of Gene Bridges, A. Francis Stewart of the Technische Universität Dresden, Rolf Müller of the Universität des Saarlandes and their colleagues took a decisive step in this direction by developing a recombineering method to directly clone entire metabolic operons.

Recombineering approaches involve the seamless recombination of a sequence of interest flanked by homologous 'arms' to any targeted location on a bacterial chromosome or plasmid. Most protocols rely on Red proteins from $\lambda$ phage to mediate recombination between a circular and linear sequence, but poor efficiency for sequences above 10 kilobases limits throughput for applications such as bioprospecting. Truncated RecE $5^{\prime}$ to 3 ' exonuclease and the RecT single-stranded annealing protein from Rac prophage can also be used but are less efficient.

To their surprise, the groups discovered that full-length RecE can mediate recombination between two linear molecules at 20 -fold greater efficiency than the best circular-linear methods, cloning up to 51 kilobases of genomic sequence into a linearized expression vector in a single reaction. They found that linear-linear recombination uses an alternate, replication-independent mechanism, and they boosted efficiency by also expressing Red $\gamma$.
The prospectors set their sites on ten gene clusters from Photorhabdus luminescens, each in a single 10-50-kilobase operon predicted to encode proteins involved in polyketide synthase and nonribosomal peptide synthesis. As is the case for many operons involved in secondary metabolism, these operons were not expressed in their native host under conditions tested in the laboratory and needed to be studied in a different host. Two of the clusters produced several new compounds in Escherichia coli.

The approach has yet to be tested on complex environmental mixtures but has the potential to unearth a wealth of compounds and medicines.

Tal Nawy

\section{RESEARCH PAPERS}

$\mathrm{Fu}$, J. et al. Full-length RecE enhances linear-linear homologous recombination and facilitates direct cloning for bioprospecting. Nat. Biotechnol. $\mathbf{3 0}$, 440-446 (2012). 